\title{
Efecto de Dosis Única Intraperitoneal de Cipermetrina en la Corteza Cerebral Somatosensorial de Ratones CF-1
}

\author{
Effect of a Single Doses Intraperitoneal of Cypermethrin \\ in Brain Somatosensory Area of Mice CF-1
}

Leonella Jiménez Venegas; Javier Quilodrán Peredo; Juan Pablo Miranda Olivares \& Héctor Rodríguez Bustos

JIMÉNEZ. V. L.; QUILODRÁN, P. J.; MIRANDA, O. J. P. \& RODRÍGUEZ, B. H. Efecto de dosis única intraperitoneal de cipermetrina en la corteza cerebral somatosensorial de ratones CF-1. Int. J. Morphol., 26(1):19-26, 2008.

RESUMEN: La cipermetrina es un pesticida ampliamente utilizado en agricultura y en salud pública. Sus efectos histopatológicos a nivel de sistema nervioso central han sido poco estudiados, aún cuando se han demostrado casos de intoxicación en humanos, con importante compromiso neurológico. El propósito de este trabajo es conocer los efectos de una dosis única intraperitoneal de cipermetrina sobre la morfología neuronal de corteza somatosensorial (láminas superficiales y profundas) de ratones CF-1. 35 ratones fueron separados en tres grupos: control $(n=5)$, control vehículo $(n=15)$ y experimental $(n=15)$; los dos últimos grupos sacrificados los días $1,8,18,26$ y 35, en grupos de a tres. Los encéfalos fueron removidos, fijados, y procesados con técnica de rutina, para un análisis morfométrico. Análisis estadístico con Kruskal Walli's test. Fueron evidenciadas alteraciones morfológicas correspondientes a muerte neuronal en todas las láminas estudiadas, a los distintos intervalos de tiempo.

PALABRAS CLAVE: Corteza somatosensorial; Apoptosis; Cipermetrina; Neurotoxicidad.

\section{INTRODUCCIÓN}

Los pesticidas ocasionan una gran toxicidad, tanto en forma profesional como accidental, en el ser humano. Un ejemplo de estos pesticidas lo constituye la cipermetrina, un piretroide sintético derivado de la planta del crisantemo (WHO, 1989).

La cipermetrina ha sido utilizada en medicina con buenos resultados en el control de la enfermedad de Chagas (Ramsey \& Schofield, 2003), en el tratamiento de la pediculosis y la escabiosis, (Cox, 2000; Nash, 2003; Heukelbach \& Feldmeier, 2004), entre otros usos (Fradin, 1998). Se han demostrado efectos deletéreos en el sistema inmunológico (Lukowicz-Ratajczak \& Krechniak, 1991a), alteraciones espermáticas (Seth et al., 2000) e incluso, actualmente algunos piretroides se estudian como uno de los posibles causales de los fenómenos neurológicos (Plapp, 1999) y reproductivos del síndrome de la Guerra del Golfo (Maconochle et al., 2004). Pero este riesgo no es sólo para las personas que manipulan el pesticida, sino también a nivel de consumidores, ya que algunos estudios evidencian elevadas concentraciones de cipermetrina en la leche de vaca (Bissacot \& Vassilieff, 1997; Sassine et al., 2004).
Investigaciones recientes alertan sobre los posibles riesgos en el desarrollo de enfermedades crónicas ante la exposición a cipermetrina, como desórdenes en el sistema nervioso debido a acumulación de pequeñas dosis en el organismo (Kolaczinski \& Curtis, 2004). Su efecto tóxico se manifiesta principalmente sobre la excitación del sistema nervioso central (SNC) y del sistema nervioso periférico (Verschoyle \& Aldrige, 1980; Krechniak \& LobodaPeplinska, 1991; McDaniel \& Moser, 1993; Crofton et al., 1995), siendo causa de intoxicaciones laborales agudas en exposiciones de corto tiempo (Chen et al., 1991), o afectando el nivel de conciencia frente a exposiciones prolongadas (He et al., 1989).

Existe escasa evidencia científica de las alteraciones neurológicas inducidas por este pesticida, que muestren cambios citológicos y las implicancias que la exposición de este tipo de productos conlleva en las células neurales, a corto y a largo plazos. Por lo que, el propósito del presente estudio es describir las alteraciones histopatológicas y morfométricas, a nivel de corteza cerebral, de tipo somatosensorial, a distintos intervalos de tiempo. 


\section{MATERIAL Y MÉTODO}

Se utilizaron 35 ratones machos cepa CF-1, de 3 meses de edad (250-300 g) del Bioterio Central de la Facultad de Medicina de la Universidad de Chile, mantenidos bajo condiciones estándar de bioterio, con comida y agua ad limitum, cuyo manejo fue aprobado por el Comité de Ética de la misma institución, siguiendo las normas del Nacional Research Council.

Se utilizó cipermetrina 92,5\% (ANASAC Chile $®)$ ), diluida en aceite vegetal de tipo comercial (Chef Maravilla (B) como vehículo. Los reactivos para técnica de rutina (hematoxilina-eosina) fueron obtenidos con un alto grado de pureza, de distintas fuentes comerciales.

Los 35 ratones fueron distribuidos en tres grupos: Control 1 (C1); no se les administró sustancia alguna $(\mathrm{n}=5)$, Control 2 (C2); se les administró vía intraperitoneal 0,2 ml. de vehículo $(\mathrm{n}=15)$ y Experimental $(\mathrm{E})$; se les administró cipermetrina $1 / 5$ de la $\mathrm{DL}_{50}$, (inyección intraperitoneal) $\left(\mathrm{DL}_{50}\right.$ en ratón es de $485 \mathrm{mg} / \mathrm{kg}$ de peso) diluido en $0,2 \mathrm{ml}$ de solución vehículo $(\mathrm{n}=15)$.

Los ratones fueron anestesiados con una dosis de ketamina al $5 \%$ (150 mg/kg vía intramuscular) y sacrificados mediante dislocación cervical. Los sacrificios en el grupo C1 se realizaron todos a las 24 horas; en los grupos $\mathrm{C} 2$ y E fueron en grupos de a tres animales, a los 1, 8, 18, 26 y 35 días.

Los encéfalos fueron removidos y fijados en una solución de formaldehído al 10\%, en buffer fosfato (pH 7,2), por 12 días, impregnados en parafina. Se obtuvieron cortes coronales seriados de 5 micrones, que fueron teñidos con hematoxilina-eosina.

Los cortes obtenidos se estudiaron, identificando el área correspondiente a la corteza cerebral somatosensorial, según Atlas de Paxinos \& Watson (1986). Se realizaron evaluaciones sobre un total de 6 secciones coronales por hemisferio, identificando las láminas superficiales (LS=LII-LIII) y profundas $(\mathrm{LP}=\mathrm{LV})$. Se cuantificó el área para morfometría, determinando porcentaje de sobrevida y densidad neuronal. Para cada grupo se obtuvieron valores promedios y desviaciones estándar, utilizando Test Kruskal Wallis, con significancia $\mathrm{p} \leq 0.05$.

La medición de neuronas vivas se realizó considerando tinción nuclear y presencia de nucleolo. Fueron consideradas neuronas muertas o degeneradas a aquellas que exhibieron una marcada eosinofilia en soma y dendrita apical. (Abdel- Rahman et al., 2004)

\section{RESULTADOS}

A) Análisis con tinción de hematoxilina-eosina en grupo control: las Figs. 1a (LS) y 1b (LP) corresponden a corteza somatosensorial de ratas del grupo $\mathrm{C} 1$ que muestran neuronas dentro de parámetros morfológicos normales.

B) Análisis con tinción de hematoxilina-eosina en el grupo experimental: En las Figs. 1c (LS) y 1d (LP) de la corteza somatosensorial, se observan, distribuidas en las distintas láminas y en diferentes proporciones, neuronas retraídas, con poca definición morfológica entre núcleo y citoplasma y con marcada tinción eosinófila, en contraste con las neuronas sobrevivientes de los mismos cortes.

C) Porcentaje de viabilidad neuronal: Al comparar los porcentajes de sobrevida entre los grupos C1 y C2 en las LS y LP, éstas no presentaron diferencias porcentuales significativas. Sin embargo, todos los grupos E evidenciaron un menor porcentaje de sobrevida con relación a los grupos $\mathrm{C} 1$ y $\mathrm{C} 2(\mathrm{p}<0,05)$, tanto en LS como LP, en todos los intervalos de tiempo (Figs. 2A y 2B).

D) Densidad neuronal: Al comparar la densidad de sobrevida neuronal en todos los intervalos de tiempo, el grupo experimental mostró una menor densidad en relación al C2, siendo estadísticamente significativa en los días 1 y 35 en LS $\mathrm{p}<0.01$ y solo al día 1 en LP $\mathrm{p}<0.02$. (Fig. 3).

\section{DISCUSIÓN}

Actualmente, son escasas las investigaciones sobre los efectos morfológicos de la cipermetrina en el sistema nervioso central, aunque los estudios existentes confirman que este insecticida produce efectos neurotóxicos difusos en neuronas de la corteza cerebral de ratas expuestas a 250 $\mathrm{mg} / \mathrm{kg}$ de $\alpha$-cipermetrina (Luty et al., 1998; Sayim et al., 2005).

Los resultados del presente trabajo muestran alteraciones morfológicas evidentes, presentes en las neuronas de la corteza somatosensorial, inducidas por cipermetrina, tanto en LS como LP, evidenciando degeneración neuronal del tipo piramidal, caracterizada por tinción eosinofílica en soma y dendritas apicales.

Investigaciones realizadas por Manna et al. (2004), con una dosis oral única de $\alpha$-cipermetrina, demostraron alteración del metabolismo hepático con un aumento del estrés oxidativo. 

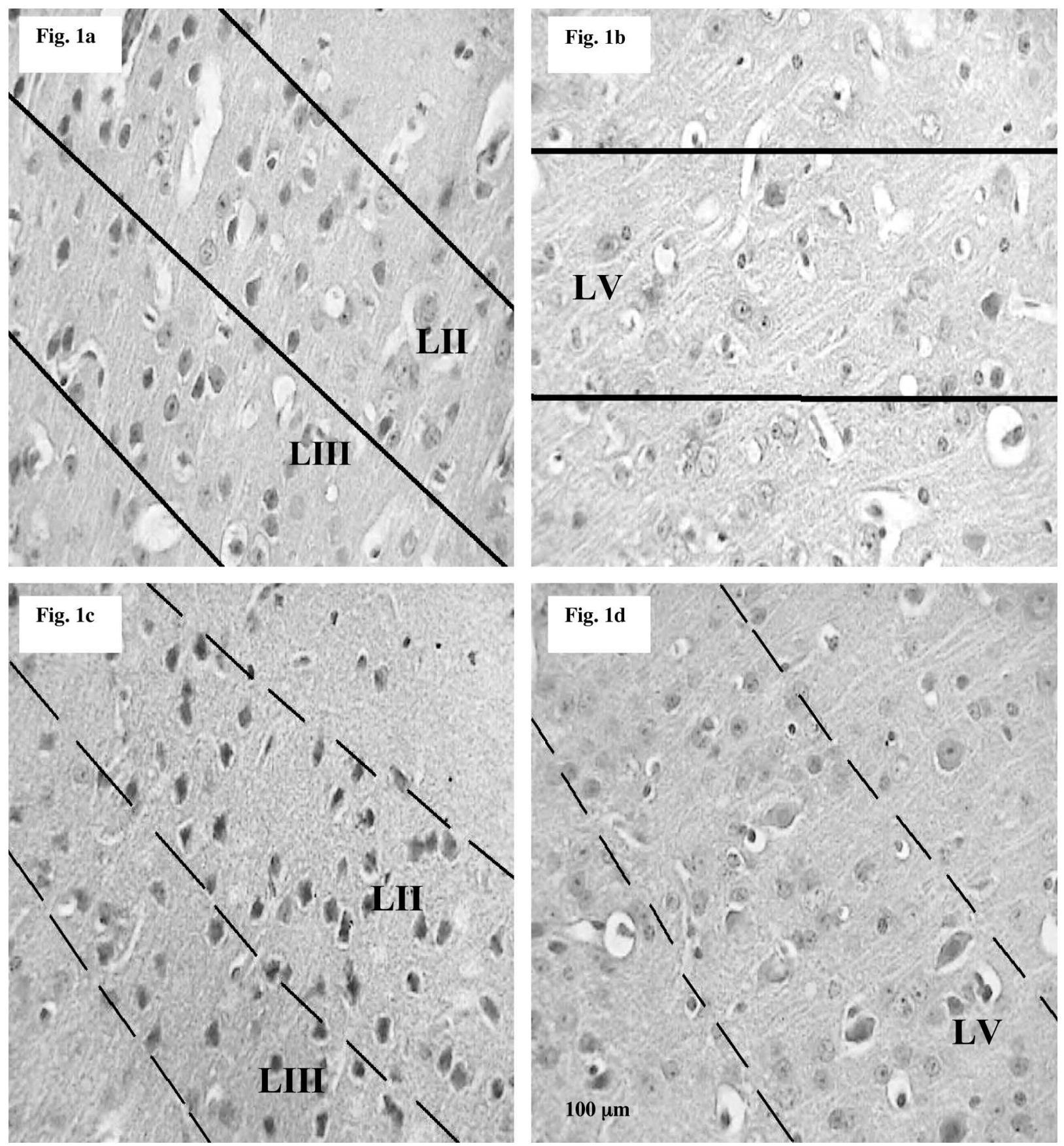

Fig. 1. Cortes coronales de corteza somatosensorial: a) ratones control: láminas superficiales; b) ratones control: láminas profundas; c) ratones grupo experimental: láminas superficiales y d) ratones grupo experimental: láminas profundas. Tinción H-E. L=lámina. Flechas indican células en proceso de muerte celular. 100x.

Por otro lado, el estrés oxidativo a nivel del sistema nervioso, producido por cipermetrina, altera el funcionamiento normal de los receptores ionotrópicos en las membranas neuronales (Eells \& Dubocovich, 1988), inhibe la actividad de la acetilcolinesterasa (Rao \& Rao, 1995), retarda el cierre de los canales de sodio voltaje-dependiente (Aldridge,
1990; Vijverberg \& van den Bercken, 1990) aumenta la liberación de acetilcolina de las terminaciones sinápticas, prolongando la depolarización por liberación de neurotransmisores que excitarían a neuronas vecinas (Eells et al., 1992). Estos acontecimientos producen un aumento aberrante en las concentraciones intracelulares de calcio, 


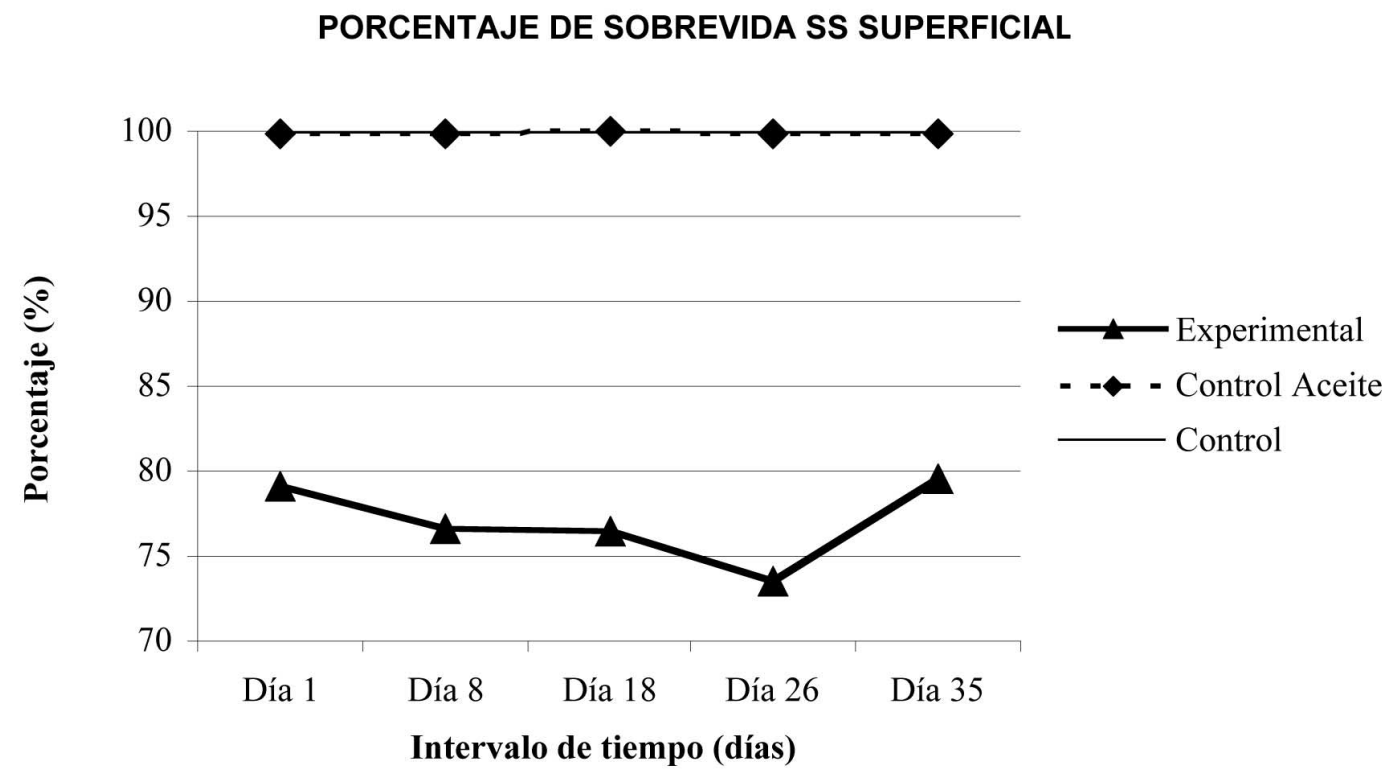

PORCENTAJE DE SOBREVIDA SS PROFUNDA

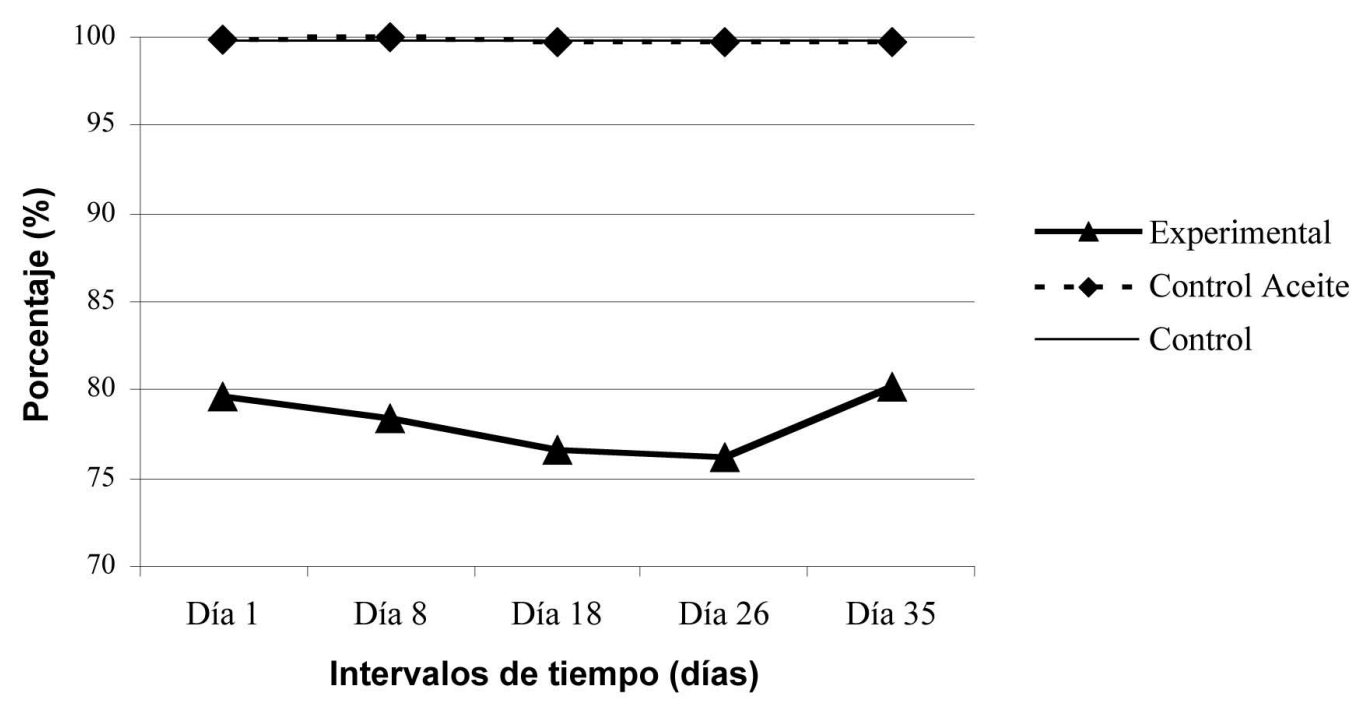

Fig. 2. Porcentaje de sobrevida neuronal de las capas superficiales (A) y profunda (B) del grupo Control, Control Aceite y Experimental, en los distintos intervalos de tiempo. $\mathrm{p}<0.05$.

como un mecanismo citotóxico que puede causar su muerte mediada por la vía de la apoptosis (Jordán, 2003); y con la consecuente alteración de su citoarquitectura con una interrupción y reducción significativa de la proteína MAP2 (Abdel-Rahman et al., 2001), mostrando una alteración morfológica y, por ende, neurodegeneración (eosinofilia en soma y dendritas).

Numerosas investigaciones en animales de laboratorio que han sido expuestos a distintas dosis de cipermetrina, han demostrado que habría repercusiones de carácter tanto agudo como crónico, en distintos niveles del organismo.

En nuestros resultados, tanto la densidad como el porcentaje de sobrevida disminuyeron en relación al grupo control. El porcentaje de sobrevida en los grupos E, con relación al C1, disminuyó en más de un $20 \%$ en todos los intervalos de tiempo, siendo el día 26 el que presentó el mayor porcentaje de muerte neuronal, tanto en LS $(26,7 \%)$ como LP $(23,4 \%)$. La tasa de sobrevida de las LS fue menor en 
Láminas superficiales LII-LIII

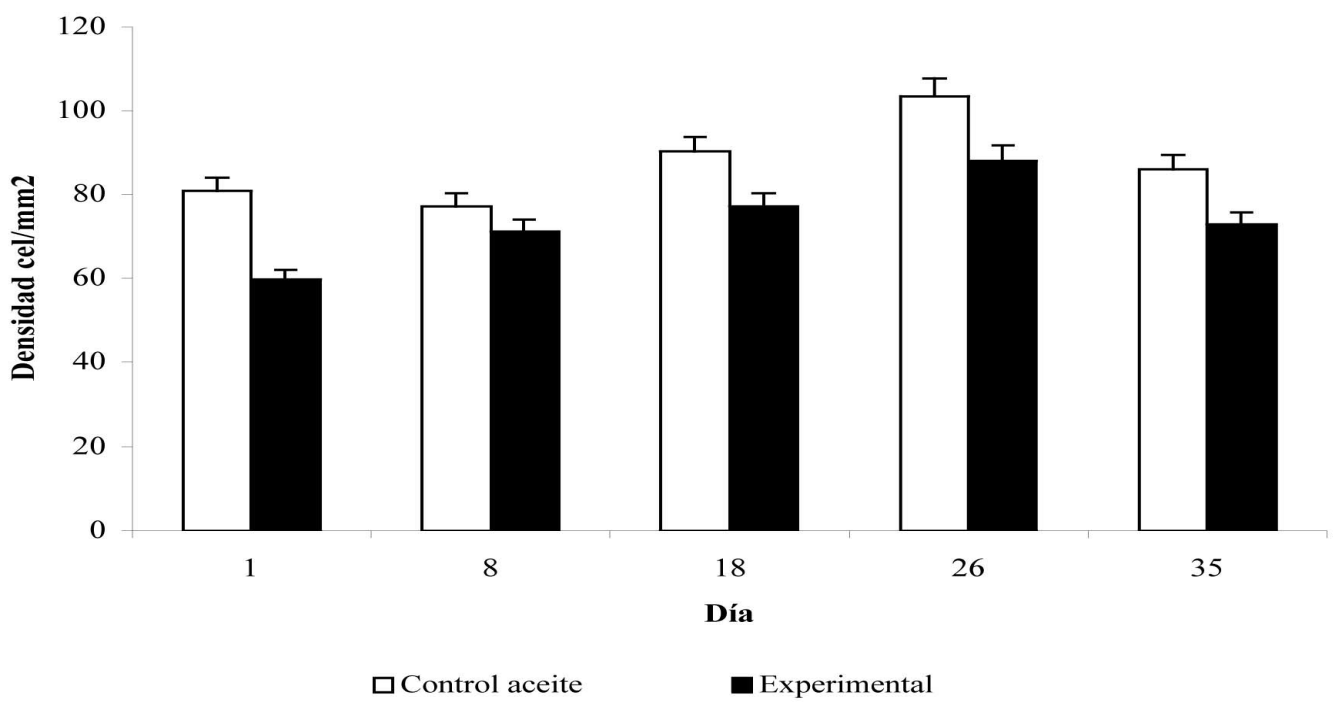

Lámina Profunda LV

120

100

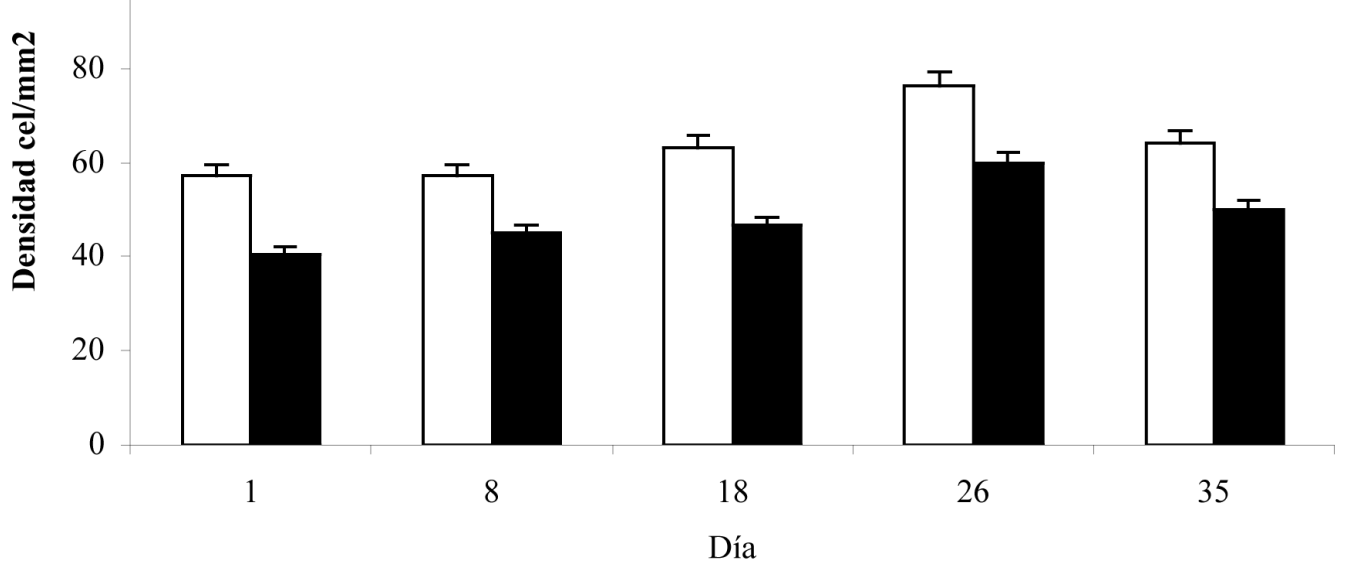

$\square$ Control aceite

Experimental

Fig. 3. Densidades de sobrevida neuronal (neuronas piramidales $/ \mathrm{mm}^{2}$ ), expresadas en promedios, de las láminas superficiales y profundas de la corteza somatosensorial de los distintos gruposestudiados para cada intervalo de tiempo. $\mathrm{p}<0.05$.

comparación con las LP, en todos los intervalos de tiempo, aunque sin diferencias significativas (Tabla I). Estos efectos se corresponderían con la alta afinidad por lípidos en el organismo que muestra la cipermetrina. El efecto agudo del día 1, estaría mediado por la acción total de la dosis de cipermetrina, en cambio la disminución creciente en los intervalos sucesivos, estaría determinado por su cinética de eliminación y su efecto residual en el tejido neural. Un $78 \%$ es eliminado a las 24 horas, $12 \%$ al octavo día y el $10 \%$ restante entre los 18-26 días. 
Tabla I. Porcentaje promedio de sobrevida neuronal según láminas e intervalos de tiempo. C1. Grupo control; C2. Grupo control aceite y E. Grupo experimental.

$$
\text { SS Láminas suerficiales SS Lámina profunda }
$$

\begin{tabular}{lccc} 
Grupo & Promedio \pm ds & Grupo & Promedio \pm ds \\
\hline C1 & $99,91 \pm 0,15$ & C1 & $99,68 \pm 0,55$ \\
C2 1 días & $99,86 \pm 0,24$ & C2 1 día & $99,89 \pm 0,19$ \\
C2 8 días & $99,85 \pm 0,27$ & C2 8 días & 100 \\
C2 18 días & 100 & C2 18 días & $99,69 \pm 0,44$ \\
C2 26 días & $99,88 \pm 0,21$ & C2 26 días & $99,73 \pm 0,47$ \\
C2 35 días & $99,91 \pm 0,16$ & C2 35 días & $99,71 \pm 0,51$ \\
E 1 día & $78,95 \pm 5,64$ & E 1 día & $79,55 \pm 1,54$ \\
E 8 días & $76,57 \pm 2,77$ & E 8 días & $78,71 \pm 5,82$ \\
E 18 días & $76,50 \pm 1,16$ & E 18 días & $76,62 \pm 0,71$ \\
E 26 días & $73,14 \pm 4,46$ & E 26 días & $76,34 \pm 3,88$ \\
E 35 días & $79,55 \pm 2,96$ & E 35 días & $80,37 \pm 2,32$
\end{tabular}

La excreción de la cipermetrina no aumenta ante exposiciones repetidas (Eadsforth et al., 1988) pero tiene implicancias en el ámbito de la toxicidad ocupacional del pesticida (Woollen et al., 1992, Heudorf \& Angerer, 2001) especialmente cuando hay combinación de piretroides (Gaughan et al., 1980), lo cual es muy importante a nivel de trabajadores del área agrícola, ya que produce neurodegeneración que podría traducirse en un futuro, en cuadros neurológicos de mayor complejidad.

JIMÉNEZ. V. L.; QUILODRÁN, P. J., MIRANDA, O. J. P. \& RODRÍGUEZ, B. H. Effects of a single doses intraperitoneal of cypermethrin in brain somatosensory area of mice CF-1. Int. J. Morphol., 26(1):19-26, 2008.

SUMMARY: The cypermethrin is a pesticide widely used in agriculture and public health. Their histopathological effects at central nervous system level, little have been studied, even though has demonstrated cases of poisoning in humans with important neurological commitment. The aim of this work is to know the effects a intraperitoneal single dose of cypermethrin on neuronal morphology of somatosensory cortex (superficial and deep laminae) of mice CF-1. 35 mice were separated in three groups: control ( $\mathrm{n}=5$ ), control vehicle $(n=15)$ and experimental $(n=15)$; the two last sacrificed groups $1,8,18,26$ and 35 days, three animals per group. The brain were removed, fixed, and process with routine technique, for a morphometric analysis. Statistical analysis with Kruskal Wallís test. We demonstrated corresponding morphologic alterations until death neuronal, in all studied laminae, at the different time intervals.

KEY WORDS: Sensory cortex; Apoptosis; Cipermethrin; Neurotoxicity.

\section{REFERENCIAS BIBLIOGRÁFICAS}

Abdel-Rahman, A.; Shetty, A. K. \& Abou-Donia, M. B. Subchronic dermal application of N,N-diethyl -toluamide (DEET) and permethrin to adult rats, alone or in combination, causes diffuse neuronal cell death and cytoskeletal abnormalities in the cerebral cortex and the hippocampus, and Purkinje neuron loss in the cerebellum. Exp. Neurol., 172(1):153-71, 2001.

Abdel-Rahman, A.; Abou-Donia, S.; El-Masry, E. \& Shetty, A. \& Abou-Donia, M. Stress and combined exposure to low doses of pyridostigmine bromide, DEET, and permethrin produce neurochemical and neuropathological alterations in cerebral cortex, hippocampus, and cerebellum. J. Toxicol. Environ. Health., A. 67(2):163-92, 2004.

Aldridge, W. N. An assesment of the toxicological properties of pyrethroids and their neurotoxicity. Crit. Rev. Toxicol., 21(2):89-104, 1990.
Bissacot, D. Z. \& Vassilieff, I. HPLC determination of flumethrin, deltamethrin, cypermethrin, and cyhalothrin residues in the milk and blood of lactating dairy cows. $J$. Anal. Toxicol., 21(5):397-402, 1997.

Chen, S. Y.; Zhang, Z. W.; He, F. S.; Yao, P. P.; Wu, Y. Q.; Sun, J. X.; Liu, L. H. \& Li, Q. G. An epidemiological study on occupational acute pyrethroid poisoning in cotton farmers. Br. J. Ind. Med., 48(2):77-81, 1991.

Cox, N. H. Permethrin treatment in scabies infestation: importance of the correct formulation. BMJ, 320(7226): 37-8, 2000.

Crofton, K. M.; Kehn, L. S. \& Gilbert, M. E. Vehicle and route dependent effects of a pyrethroid insecticide, deltamethrin, on motor function in the rat. Neurotoxicol. Teratol., 17(4):489-95. 1995. 
Eadsforth, C. V.; Bragt, P. C. \& van Sittert, N. J. Human dose-excretion studies with pyrethroid insecticides cypermethrin and alphacypermethrin: relevance for biological monitoring. Xenobiotica, 18(5):603-14, 1988.

Eells, J. T.; Bandettini, P. A.; Holman, P. A. \& Propp, J. M. Pyrethroid insecticide-induce alterations in ammalian synaptic membrane potential. J. Pharmacol. Exp. Ther, 262(3):1173-81, 1992.

Eells, J. T. \& Dubocovich, M. L. Pyrethroid insecticides evoke neurotransmitter release from rabbit striatal slices. J. Pharmacol. Exp. Ther., 264(2):514-21, 1988.

Fradin, M. S. Mosquitoes and mosquito repellents: a clinician's guide. Ann. Intern. Med., 128(11):931-40, 1998.

Gaughan, L. C.; Engel, J. L. \& Casida, J. E. Pesticide interactions: Effects of organophosphorus pesticidas on the metabolism, toxicity, and persistence of selected pyrethroid insecticides. Pestic. Biochem. Physiol., 14: 81-5, 1980.

He, F.; Wang, S.; Liu, L.; Chen, S.; Zhang Z, Sun J. Clinical manifestations and diagnosis of acute pyrethroid poisoning. Arch. Toxicol., 63(1):54-8, 1989.

Heudorf, U. \& Angerer, J. Metabolites of pyrethroids insecticides in urine specimens: current exposure in an urban population in Germany. Environ Health Perspect., 109(3):213-7, 2001.

Heukelbach, J. \& Feldmeier, H. Ectoparasites the underestimated realm. Lancet, 13(363):889-91, 2004.

Jordán, J. Apoptosis: muerte celular programada. OFFARM 22(6):100-6, 2003

Kolaczinski, J. H. \& Curtis, C. F. Chronic illnes as a result of low-level exposure to synthetic pyrethroid insecticides: a review of the debate. Food Chem. Toxicol., 42(5):697-706, 2004.

Krechniak, J. \& Loboda-Peplinska, T. Effect of selected pyrethroids on cholinesterase activity in rat. Bromatol. Chem. Toksykol., 24:205-8, 1991.

Lukowicz-Ratajczak, J. \& Krechniak, J. Effect of decamethrin on immunological system. Part I. Immuno-suppresive action. Bromatol. Chem. Toksykol., 24(1): 57-61, 1991a.
Luty, S.; Latuszynska, J.; Halliop, J.; Tochman, A.; Obuchowska, D.; Przylepa, E. \& Korczak, E. Toxicity of dermally applied alpha-cymermethrin in rats. Ann. Agric. Environ. Med., 5:109-16, 1998.

Maconochle, N.; Doyle, P. \& Carson, C. Infertility among male UK veterans of the 1990-1 Gulf war: reproductive cohort study. BMJ, 329:196-201, 2004.

Manna, S.; Bhattacharyya, D.; Basak, D. K. \& Mandal, T. K. Single oral dose toxicity study of a-cypermethrin in rats. Indian J. Pharmacol., 36(1):25-8, 2004.

McDaniel, K. L. \& Moser, V. C. Utility of a neurobehavioral screening battery for differentiating the effects of two pyrethroids, permethrin and cypermethrin. Neurotoxicol. Teratol., 15(2):71-83, 1993.

Nash, B. Treating head lice. BMJ, 326:1256-7, 2003.

Paxinos, G. \& Watson, C. The rat brain in stereotaxic coordinates. $2^{\text {th }}$. Edition. Academic Press Inc, New York, 1986.

Plapp, F. W. Jr. Permethrin and the Gulf War Syndrome. Arch. Environ Health, 54 (5):312, 1999.

Ramsey. J. M. \& Schofield, C. J. Control of Chagas disease vectors. Salud Pública Mex., 45(2):123-8, 2003.

Rao, G. V. \& Rao, K. S. Modulation in acetylcholinesterase of rat brain by pyrethroids in vivo and in vitro kinetic study. J. Neurochem., 65(5):2259-66, 1995.

Sassine, A.; Moura, S.; Leo, V. M. \& Bustillos O. V. Cypermethrin residues determination in the milk of a lactating dairy cow by gas chromatography-ion trap mass spectorometry. J. Ann. Toxicology, 28(4):238-41, 2004.

Sayim, F.; Yavasoglu, N. U. K.; Uyanikgil, Y.; Aktug, H.; Yavasoglu, A. \& Turgut, M. Neurotoxic effects of cypermethrin in Wistar rats: a haematological, biochemical and histopathological study. J. Health Sci., 51(3):300-7, 2005.

Seth, P. K.; Jaffery, F. N. \& Khanna, V. K. Toxicology. Indian J. Pharmacol., 32:S134-51, 2000.

Verschoyle, R. D. \& Aldrige, W. N. Structure-activity relationship of some pyrethroids in rats. Arch. Toxicol., 45(4):325-9, 1980. 
JIMÉNEZ. V. L.; QUILODRÁN, P. J., MIRANDA, O. J. P. \& RODRÍGUEZ, B. H. Efecto de dosis única intraperitoneal de cipermetrina en la corteza cerebral somatosensorial de ratones CF-1. Int. J. Morphol., 26(1):19-26, 2008.

Vijverberg, H. P. \& van den Bercken, J. Neurotoxicological effects and the mode of action of pyrethroid insecticides. Crit. Rev. Toxicol., 21(2):105-26, 1990.

Woollen, B. H.; Marsh, J. R.; Laird, W. J. \& Lesser, J. E. The metabolism of cypermethrin in man: differences in urinary metabolite profiles following oral and dermal administration. Xenobiotica, 22(8):983-91, 1992.

World Health Organization (WHO). Environmental Health Criteria. Cypermethrin. Geneva: United Nations Environment Programme, the International Labour Organization, and the World Health Organization, 82, 1989.
Dirección para correspondencia:

Prof. Leonella Jiménez Venegas

Programa de Anatomía y Biología del Desarrollo Instituto de Ciencias Biomédicas.

Facultad de Medicina

Universidad de Chile.

Santiago - CHILE

Fono-fax: 56-2-9786091

56-2-9786264

Email: ljimenez@med.uchile.cl

Recibido : 16-08-2007

Aceptado: 22-11-2007 